UFIFT-HEP-97-28, UPR-0773-T

hep-ph/9711339

November 1997

\title{
On the Anomalous $U(1)$ in Free Fermionic Superstring Models
}

\author{
Gerald B. Cleaver ${ }^{1}$ and Alon E. Faraggi蚂 \\ ${ }^{1}$ Department of Physics and Astronomy \\ University of Pennsylvania \\ Philadelphia, PA 19104, USA \\ 2 Institute for Fundamental Theory, \\ Department of Physics, \\ University of Florida, \\ Gainesville, FL 32611, USA
}

\begin{abstract}
The realistic free fermionic models have had an intriguing success in explaining different properties of the observed particle spectrum. In this paper we discuss in some detail the anomalous $U(1)$ symmetry which exists in these models. We study the properties of the anomalous $U(1)$ in both the more realistic NAHE-based free fermionic models and those in a general NAHE class. Appearance of an anomalous $U(1)$ in the more realistic NAHE models is shown to be an effect of reduction of world-sheet supersymmetry from $(2,2)$ to $(2,0)$. We show, however, that in more general $(2,1)$ and $(2,0)$ models, all $U(1)$ can remain anomaly-free under certain conditions. Several phenomenological issues related to the anomalous $U(1)$ are discussed. In particular, we note that in some examples the anomalous $U(1)$ arises from the breaking $E_{6} \rightarrow S O(10) \times U(1)_{A}$, resulting in $U(1)_{A}$ being family universal.
\end{abstract}

\footnotetext{
*gcleaver@langacker.hep.upenn.edu

†faraggi@phys.ufl.edu
} 


\section{Introduction}

The more realistic superstring models in the free fermionic class have had intriguing success in providing plausible explanations for various properties of the Standard Model [1]. A few of these include: the natural emergence of three generations; the origin of the heavy top quark mass; the qualitative pattern of the fermion mass spectrum; the stability of the proton; and more [1].

A general property of these models, which is also shared by many other superstring vacua, is the existence of an "anomalous" $U(1)$. The presence of an Abelian "anomalous" symmetry in superstring derived models yields many desirable phenomenological implications from the point of view of the effective low energy field theory. The list of a few of those possibilities include: Generation of a Fayet-Iliopoulos $D$-term [2]; Breaking and rank reduction of the four dimensional gauge group [3]; Generation of the fermion mass hierarchy [4, 5, 6]; Predicting neutrino mixing patterns [7]; Implication of the relation between anomaly cancelation and gauge coupling normalization on the weak mixing angle [8]; Anomalous $U(1)$ as the trigger of supersymmetry breaking [9, 10, 11]; Cosmological implications of the anomalous $U(1)$ such as implications for inflation and cosmic strings [12]; Anomalous $U(1)$ in connection with the strong CP problem [13]; and Non-perturbative considerations of the anomalous $U(1)$ [14].

These phenomenological implications make evident the need to determine what we may actually learn from the anomalous $U(1)$ symmetries appearing in realistic superstring models. In this paper we undertake this task by examining the anomalous $U(1)$ in the NAHE-based [15, 16, 17] free fermionic models. We first discuss the origin of the anomalous $U(1)$ and classify the different sources that may contribute to the anomaly. Further, we discuss general constraints on a $U(1)$ becoming anomalous and properties of a model that prevent the appearance of the anomaly. We then proceed to examine the anomalous $U(1)$ in specific models and discuss some of the phenomenological implications on the final gauge group and on supersymmetry breaking. An important property of the anomalous $U(1)$ in some of the free fermionic models is the fact that it is family universal. In the NAHE-based models, the origin of this universality arises due to the cyclic permutation symmetry that characterizes the $Z_{2} \times Z_{2}$ orbifold, which underlies these realistic free fermionic models. The contribution to the anomalous $U(1)$ from sectors belonging to the $Z_{2} \times Z_{2}$ orbifold is family universal. This property is often destroyed when additional basis vectors, beyond those that correspond to the $Z_{2} \times Z_{2}$ orbifold, are added to obtain the full set of basis vectors necessary for these three generation models. However, in some models the anomalous $U(1)$ universality is preserved and may serve as an additional phenomenological criteria in the classification of this class of models. 


\section{Realistic free fermionic models}

In this section we recapitulate the main structure of the free fermionic models. We discuss here the main features which are important for understanding the origin or the anomalous $U(1)$ and for eventually relating the phenomenological implications of the anomalous $U(1)$ in these models to the basic structures underlying the models.

In the free fermionic formulation of the heterotic string [18] all the degrees of freedom needed to cancel the conformal anomaly are represented in terms of internal free fermions propagating on the string world-sheet. In four dimensions, this requires 20 left-moving and 44 right-moving real world-sheet fermions. Equivalently, some real fermions may be paired to form complex fermions. Under parallel transport around a noncontractible loop, the fermionic states pick up a phase. Specification of the phases for all world-sheet fermions around all noncontractible loops contributes to the spin structure of the model. Such spin structures are usually given in the form of boundary condition "vectors", with each element of the vector specifying the phase of a corresponding world-sheet fermion. The possible spin structures are constrained by string consistency requirements (e.g. modular invariance). A model is constructed by choosing a set of boundary condition basis vectors, which satisfies the modular invariance constraints. The basis vectors, $\mathbf{b}_{k}$, span a finite additive group $\Xi=\sum_{k} n_{k} \mathbf{b}_{k}$ where $n_{k}=0, \cdots, N_{z_{k}}-1$. The physical massless states in the Hilbert space of a given sector $\alpha \in \Xi$, are obtained by acting on the vacuum with bosonic and fermionic operators and by applying the generalized GSO projections. The $U(1)$ charges, $Q(f)$, with respect to the unbroken Cartan generators of the four dimensional gauge group, which are in one-to-one correspondence with the $U(1)$ currents $f^{*} f$ for each complex fermion $f$, are given by:

$$
Q(f)=\frac{1}{2} \alpha(f)+F(f)
$$

where $\alpha(f)$ is the boundary condition of the world-sheet fermion $f$ in the sector $\alpha$, and $F_{\alpha}(f)$ is a fermion number operator counting each mode of $f$ once (and if $f$ is complex, $f^{*}$ minus once). For periodic fermions, $\alpha(f)=1$ and the vacuum is a spinor in order to represent the Clifford algebra of the corresponding zero modes. For each periodic complex fermion $f$ there are two degenerate vacua $|+\rangle,|-\rangle$, annihilated by the zero modes $f_{0}$ and $f_{0}{ }^{*}$ and with fermion numbers $F(f)=0,-1$, respectively.

\subsection{The NAHE set}

The boundary condition basis vectors which generate the realistic free fermionic models under discussion are, in general, divided into two major subsets. The first subset consists of the NAHE set [15, 16, 17], which is a set of five boundary condition basis vectors denoted $\left\{\mathbf{1}, \mathbf{S}, \mathbf{b}_{1}, \mathbf{b}_{2}, \mathbf{b}_{3}\right\}$. With ' 0 ' indicating Neveu-Schwarz boundary conditions and ' 1 ' indicating Ramond boundary conditions, these vectors are as 
follows:

\begin{tabular}{c|c|ccc|c|ccc|c} 
& $\psi^{\mu}$ & $\chi^{12}$ & $\chi^{34}$ & $\chi^{56}$ & $\bar{\psi}^{1, \ldots, 5}$ & $\bar{\eta}^{1}$ & $\bar{\eta}^{2}$ & $\bar{\eta}^{3}$ & $\bar{\phi}^{1, \ldots, 8}$ \\
\hline \hline $\mathbf{1}$ & 1 & 1 & 1 & 1 & $1, \ldots, 1$ & 1 & 1 & 1 & $1, \ldots, 1$ \\
$\mathbf{S}$ & 1 & 1 & 1 & 1 & $0, \ldots, 0$ & 0 & 0 & 0 & $0, \ldots, 0$ \\
\hline $\mathbf{b}_{1}$ & 1 & 1 & 0 & 0 & $1, \ldots, 1$ & 1 & 0 & 0 & $0, \ldots, 0$ \\
$\mathbf{b}_{2}$ & 1 & 0 & 1 & 0 & $1, \ldots, 1$ & 0 & 1 & 0 & $0, \ldots, 0$ \\
$\mathbf{b}_{3}$ & 1 & 0 & 0 & 1 & $1, \ldots, 1$ & 0 & 0 & 1 & $0, \ldots, 0$
\end{tabular}

\begin{tabular}{c|cc|cc|cc} 
& $y^{3, \ldots, 6}$ & $\bar{y}^{3, \ldots, 6}$ & $y^{1,2}, \omega^{5,6}$ & $\bar{y}^{1,2}, \bar{\omega}^{5,6}$ & $\omega^{1, \ldots, 4}$ & $\bar{\omega}^{1, \ldots, 4}$ \\
\hline \hline $\mathbf{1}$ & $1, \ldots, 1$ & $1, \ldots, 1$ & $1, \ldots, 1$ & $1, \ldots, 1$ & $1, \ldots, 1$ & $1, \ldots, 1$ \\
$\mathbf{S}$ & $0, \ldots, 0$ & $0, \ldots, 0$ & $0, \ldots, 0$ & $0, \ldots, 0$ & $0, \ldots, 0$ & $0, \ldots, 0$ \\
\hline $\mathbf{b}_{1}$ & $1, \ldots, 1$ & $1, \ldots, 1$ & $0, \ldots, 0$ & $0, \ldots, 0$ & $0, \ldots, 0$ & $0, \ldots, 0$ \\
$\mathbf{b}_{2}$ & $0, \ldots, 0$ & $0, \ldots, 0$ & $1, \ldots, 1$ & $1, \ldots, 1$ & $0, \ldots, 0$ & $0, \ldots, 0$ \\
$\mathbf{b}_{3}$ & $0, \ldots, 0$ & $0, \ldots, 0$ & $0, \ldots, 0$ & $0, \ldots, 0$ & $1, \ldots, 1$ & $1, \ldots, 1$
\end{tabular}

with the following choice of phases which define how the generalized GSO projections are to be performed in each sector of the theory:

$$
C\left(\begin{array}{l}
\mathbf{b}_{i} \\
\mathbf{b}_{j}
\end{array}\right)=C\left(\begin{array}{c}
\mathbf{b}_{i} \\
\mathbf{S}
\end{array}\right)=C\left(\begin{array}{l}
\mathbf{1} \\
\mathbf{1}
\end{array}\right)=-1 .
$$

The remaining projection phases can be determined from those above through the self-consistency constraints. The precise rules governing the choices of such vectors and phases, as well as the procedures for generating the corresponding space-time particle spectrum, are given in Refs. [19].

The basis vector $\mathbf{S}$ generates the space-time supersymmetry. The set of basis vectors $\{\mathbf{1}, \mathbf{S}\}$ generates a model with $N=4$ space-time supersymmetry and $S O(44)$ gauge group in the right-moving sector. Imposing the GSO projections of the basis vectors $\mathbf{b}_{1}, \mathbf{b}_{2}$ and $\mathbf{b}_{3}$ reduces the $N=4$ supersymmetry to $N=1$ and breaks the gauge group to $S O(10) \times S O(6)^{3} \times E_{8}$. The space-time vector bosons that generate the gauge group arise from the Neveu-Schwarz sector and from the sector $\mathbf{I} \equiv \mathbf{1}+\mathbf{b}_{1}+\mathbf{b}_{2}+\mathbf{b}_{3}$. The Neveu-Schwarz sector produces the generators of $S O(10) \times$ $S O(6)^{3} \times S O(16)$, while the sector $\mathbf{1}+\mathbf{b}_{1}+\mathbf{b}_{2}+\mathbf{b}_{3}$ produces the spinorial $\mathbf{1 2 8}$ of $S O(16)$ and completes the hidden gauge group to $E_{8}$. The three basis vectors $\mathbf{b}_{1}, \mathbf{b}_{2}$ and $\mathbf{b}_{3}$ correspond to the three twisted sectors of the $Z_{2} \times Z_{2}$ orbifold. Each one of these sectors produces 16 multiplets in the $\mathbf{1 6}$ representation of $S O(10)$.

While the realistic free fermionic models have $(2,0)$ world-sheet supersymmetry [20], the NAHE set by itself produces a model with $(2,1)$ world-sheet supersymmetry. The NAHE set belongs to a small class of heterotic string models that correspond to type-II models under the $H$-map [21]. Consistent type-II strings have at least $(1,1)$ world-sheet supersymmetry and at least $(2,1)$ world-sheet supersymmetry if they also possess space-time supersymmetry. World-sheet supersymmetry is preserved 
under the $H$-map. Relatedly, the $H$-map and the associated $(2,1)$ or $(2,2)$ worldsheet supersymmetry reveal that the twelve internal left- and right-moving fermions, $\{y, \omega\}$ and $\{\bar{y}, \bar{\omega}\}$, correspond to the internal six-dimensional compactified torus in the bosonic formulation, while $\{\chi\}$ and $\{\bar{\eta}\}$ are their respective left- and right-moving complexified world-sheet superpartners. The boundary conditions of all of these fields are unaffected by the $H$-map. If a heterotic string has at least an $S O(10) \times E_{8}$ gauge group (and $N=2$ left-moving world-sheet supersymmetry), then it is mapped to a $(2,1)$ type-II sting, whereas a heterotic string with at least an $E_{6} \times E_{8}$ gauge group is identified with a $(2,2)$ type-II string. Further, a $E_{7} \times E_{8}$ gauge group is mapped to a $(2,2+4)$ model, while $E_{8} \times E_{8}$ corresponds to a model with $(2,2+2+2)$ [22].

The correspondence of the NAHE set with a $Z_{2} \times Z_{2}$ orbifold is illustrated by adding to the NAHE the boundary condition basis vector $\mathbf{X}$ with periodic boundary conditions for the world-sheet fermions $\left\{\bar{\psi}^{1, \cdots, 5}, \bar{\eta}^{1,2,3}\right\}$, and antiperiodic boundary conditions for all others,

$$
\mathbf{X}=(0, \cdots, 0 \mid \underbrace{1, \cdots, 1}_{\psi^{1, \cdots, 5}, \eta^{1,2,3}}, 0, \cdots, 0) .
$$

The choice of generalized GSO projection coefficients is

$$
C\left(\begin{array}{l}
\mathbf{X} \\
\mathbf{b}_{j}
\end{array}\right)=-C\left(\begin{array}{c}
\mathbf{X} \\
\mathbf{S}
\end{array}\right)=C\left(\begin{array}{c}
\mathbf{X} \\
\mathbf{1}
\end{array}\right)=+1
$$

With the set $\left\{\mathbf{1}, \mathbf{S}, \mathbf{b}_{1}, \mathbf{b}_{2}, \mathbf{b}_{3}, \mathbf{X}\right\}$ the gauge group is $E_{6} \times U(1)^{2} \times S O(4)^{3} \times E_{8}$. (Therefore the model clearly has $(2,2)$ world-sheet supersymmetry). For our purposes here it is more convenient to generate the same model with the basis vectors

$$
\left\{\mathbf{1}, \mathbf{S}, \xi=\mathbf{1}+\mathbf{b}_{1}+\mathbf{b}_{2}+\mathbf{b}_{3}, \mathbf{X}, \mathbf{b}_{1}, \mathbf{b}_{2}\right\}
$$

and the generalized GSO projection coefficients

$$
c\left(\begin{array}{c}
\mathbf{b}_{i} \\
\mathbf{b}_{j}
\end{array}\right)=c\left(\begin{array}{c}
\mathbf{b}_{i} \\
\mathbf{S}
\end{array}\right)=c\left(\begin{array}{c}
\mathbf{b}_{i} \\
\xi, \mathbf{X}
\end{array}\right)=-c\left(\begin{array}{l}
\xi, \mathbf{X} \\
\xi, \mathbf{X}
\end{array}\right)=-c\left(\begin{array}{l}
\mathbf{1} \\
\mathbf{1}
\end{array}\right)=-1 .
$$

The first four vectors in the basis $\{\mathbf{1}, \mathbf{S}, \xi, \mathbf{X}\}$ generate a model with $N=4$ spacetime supersymmetry with an $E_{8} \times S O(12) \times E_{8}$ gauge group. The sector $\mathbf{S}$ generates $N=4$ space-time supersymmetry. The first and second $E_{8}$ are obtained from the world-sheet fermionic states $\left\{\bar{\psi}^{1, \cdots, 5}, \bar{\eta}^{1,2,3}\right\}$ and $\left\{\bar{\phi}^{1, \cdots, 8}\right\}$, respectively, while $S O(12)$ is obtained from $\{\bar{y}, \bar{\omega}\}^{1, \cdots, 6}$. The Neveu-Schwarz sector produces the adjoint representations of $S O(16) \times S O(12) \times S O(16)$. The sectors $\mathbf{X}$ and $\xi$ produce the spinorial representation of $S O(16)$ of the observable and hidden sectors respectively, and complete the observable and hidden gauge groups to $E_{8} \times E_{8}$.

The vectors $\mathbf{b}_{1}$ and $\mathbf{b}_{2}$ break the gauge symmetry to $E_{6} \times U(1)^{2} \times S O(4)^{3} \times E_{8}$ and $N=4$ to $N=1$ space-time supersymmetry. We denote the $U(1)$ generators produced 
by the world-sheet currents : $\bar{\eta}^{i *} \bar{\eta}^{i}$ : as $U(1)_{i}$. The fermionic states $\left\{\chi^{12}, \chi^{34}, \chi^{56}\right\}$ and $\left\{\bar{\eta}^{1}, \bar{\eta}^{2}, \bar{\eta}^{3}\right\}$ give the usual "standard-embedding", with $b\left(\chi^{12}, \chi^{34}, \chi^{56}\right)=b\left(\bar{\eta}^{1}, \bar{\eta}^{2}, \bar{\eta}^{3}\right)$. The $U(1)$ current of the left-moving $N=2$ world-sheet supersymmetry is given by

$$
J(z)=\sum_{i=(12),(34),(56)}: \chi^{i *} \chi^{i}:
$$

The $U(1)_{i}$ charge combination appearing in the decomposition of $E_{6}$ under $S O(10) \times$ $U(1)$ is given by

$$
U(1)_{E_{6}}=U(1)_{1}+U(1)_{2}+U(1)_{3},
$$

while the charges of the two orthogonal combinations are specified by

$$
\begin{aligned}
& U(1)^{\prime}=U(1)_{1}-U(1)_{2} \\
& U(1)^{\prime \prime}=U(1)_{1}+U(1)_{2}-2 U(1)_{3} .
\end{aligned}
$$

The three $S O(4)$ gauge groups are produced by the right-moving world-sheet fermionic fields $\left\{\bar{y}^{3, \cdots, 6}\right\},\left\{\bar{y}^{1}, \bar{y}^{2} \bar{\omega}^{5}, \bar{\omega}^{6}\right\}$ and $\left\{\bar{\omega}^{1, \cdots, 4}\right\}$.

The sectors $\left(\mathbf{b}_{1} ; \mathbf{b}_{1}+\mathbf{X}\right),\left(\mathbf{b}_{2} ; \mathbf{b}_{2}+\mathbf{X}\right)$ and $\left(\mathbf{b}_{3} ; \mathbf{b}_{3}+\mathbf{X}\right)$ each give eight 27's of $E_{6}$. The $(N S ; N S+\mathbf{X})$ sector gives in addition to the vector bosons and spin two states, three copies of scalar representations in $\mathbf{2 7}+\overline{\mathbf{2 7}}$ of $E_{6}$. The net number of generations is 24 in the 27 representation of $E_{6}$. The same model is constructed in the orbifold formulation [23] by first constructing the Narain lattice [24] with $S O(12) \times E_{8} \times E_{8}$ gauge group $N=4$ supersymmetry. The gauge is then broken to $E_{6} \times U(1)^{2} \times S O(4)^{3} \times E_{8}$ after applying the $Z_{2} \times Z_{2}$ twisting $S O(12)$ lattice and the three twisted sector produce 48 fixed points, which correspond to the 24 generations in the fermionic construction [25].

\subsection{Beyond the NAHE set}

At the level of the NAHE set the observable gauge group is $S O(10) \times S O(6)^{3}$ and the number of generations is 48 , sixteen from each sector $\mathbf{b}_{1}, \mathbf{b}_{2}$ and $\mathbf{b}_{3}$. The $S O(6)^{3}$ symmetries are horizontal flavor dependent symmetries. To break the gauge group to the standard model and to reduce the number of generations, we must add additional boundary condition basis vectors to the NAHE set. These additional basis vectors break the $S O(10)$ and the flavor $S O(6)$ gauge symmetries and in turn reduce the number of generations to three. In the process, world-sheet supersymmetry is reduced from $(2,1)$ to $(2,0)$ via basis vectors like $\gamma$ shown below. $(2,1)$ is broken because the $\{\eta, y, \omega\}$ components of gamma do not correspond to a symmetry (mod change of sign) of the right-moving supercurrent

$$
\begin{aligned}
\bar{T}_{3 / 2} & =\bar{T}_{3 / 2}^{+1}+\bar{T}_{3 / 2}^{-1} \\
& =\sum_{i=1}^{3} \frac{1}{\sqrt{2}}\left(\bar{\eta}^{i}+\bar{\eta}^{i *}\right) \bar{y}^{2 i-1} \bar{\omega}^{2 i-1}+\sum_{i=1}^{3} \frac{-i}{\sqrt{2}}\left(\bar{\eta}^{i}-\bar{\eta}^{i *}\right) \bar{y}^{2 i} \bar{\omega}^{2 i},
\end{aligned}
$$


within the right-moving $N=1$ world-sheet supersymmetry. $\bar{T}_{3 / 2}^{+1}$ and $\bar{T}_{3 / 2}^{-1}$ are the two supercurrents within $N=2$. Note, however, that $2 \gamma$ is consistent with $N=1$.

The breaking of the gauge group and the reduction to three generations are done simultaneously. In fact the reduction to three generations is correlated with the breaking of the flavor $S O(6)^{3}$ symmetries to a product of horizontal $U(1)$ symmetries. The appealing property of these NAHE-based free fermionic models is that the emergence of three generations is correlated with the underlying $Z_{2} \times Z_{2}$ orbifold structure. While three generations can appear from other structures [26], here each generation is obtained from one of the twisted sectors of the $Z_{2} \times Z_{2}$ orbifold. Table (2.14) is an example of a choice of additional boundary condition basis vectors which produce a three generation model with $S U(3) \times S U(2) \times U(1)^{2}$ gauge group. The complete spectrum and charges under the four dimensional gauge group are given in ref. [27].

\begin{tabular}{c|c|cccc|ccc|ccccc|ccccccc} 
& $\psi^{\mu}$ & $\chi^{12}$ & $\chi^{34}$ & $\chi^{56}$ & \multicolumn{4}{|c|}{$\bar{\psi}^{1, \ldots, 5}$} & $\bar{\eta}^{1}$ & $\bar{\eta}^{2}$ & $\bar{\eta}^{3}$ & \multicolumn{4}{|c}{$\bar{\phi}^{1, \ldots, 8}$} & \\
\hline \hline$\alpha$ & 0 & 0 & 0 & 0 & 1 & 1 & 1 & 0 & 0 & 0 & 0 & 0 & 1 & 1 & 1 & 1 & 0 & 0 & 0 & 0 \\
$\beta$ & 0 & 0 & 0 & 0 & 1 & 1 & 1 & 0 & 0 & 0 & 0 & 0 & 1 & 1 & 1 & 1 & 0 & 0 & 0 & 0 \\
$\gamma$ & 0 & 0 & 0 & 0 & $\frac{1}{2}$ & $\frac{1}{2}$ & $\frac{1}{2}$ & $\frac{1}{2}$ & $\frac{1}{2}$ & $\frac{1}{2}$ & $\frac{1}{2}$ & $\frac{1}{2}$ & $\frac{1}{2}$ & 0 & 1 & 1 & $\frac{1}{2}$ & $\frac{1}{2}$ & $\frac{1}{2}$ & 0 \\
$2 \gamma$ & 0 & 0 & 0 & 0 & 1 & 1 & 1 & 1 & 1 & 1 & 1 & 1 & 1 & 0 & 0 & 0 & 1 & 1 & 1 & 0
\end{tabular}

\begin{tabular}{c|cccc|cccc|cccc} 
& $y^{3} y^{6}$ & $y^{4} \bar{y}^{4}$ & $y^{5} \bar{y}^{5}$ & $\bar{y}^{3} \bar{y}^{6}$ & \multicolumn{2}{|c|}{$y^{1} \omega^{5}$} & $y^{2} \bar{y}^{2}$ & $\omega^{6} \bar{\omega}^{6}$ & $\bar{y}^{1} \bar{\omega}^{5}$ & $\omega^{2} \omega^{4}$ & $\omega^{1} \bar{\omega}^{1}$ & $\omega^{3} \bar{\omega}^{3} \bar{\omega}^{2} \bar{\omega}^{4}$ \\
\hline \hline$\alpha$ & 1 & 0 & 0 & 0 & 0 & 0 & 1 & 1 & 0 & 0 & 1 & 1 \\
$\beta$ & 0 & 0 & 1 & 1 & 1 & 0 & 0 & 0 & 0 & 1 & 0 & 1 \\
$\gamma$ & 0 & 1 & 0 & 1 & 0 & 1 & 0 & 1 & 1 & 0 & 0 & 0 \\
$2 \gamma$ & 0 & 0 & 0 & 0 & 0 & 0 & 0 & 0 & 0 & 0 & 0 & 0
\end{tabular}

with the choice of GSO projection coefficients:

$$
c\left(\begin{array}{c}
\mathbf{b}_{j} \\
\alpha, \beta, \gamma
\end{array}\right)=-c\left(\begin{array}{l}
\alpha \\
\mathbf{1}
\end{array}\right)=c\left(\begin{array}{l}
\alpha \\
\beta
\end{array}\right)=-c\left(\begin{array}{c}
\beta \\
\mathbf{1}
\end{array}\right)=c\left(\begin{array}{c}
\gamma \\
\mathbf{1}, \alpha
\end{array}\right)=-c\left(\begin{array}{l}
\gamma \\
\beta
\end{array}\right)=-1
$$

$(\mathrm{j}=1,2,3)$, with the others specified by modular invariance and space-time supersymmetry. We comment that the vector $2 \gamma$ plays a particularly important role in understanding the origin of the anomalous $U(1)$ in the three generation free fermionic models, as will be discussed further below.

\section{$2.3(2,2) \rightarrow(2,1) \rightarrow(2,0)$ models}

The vector $\mathbf{X}$ combined with the NAHE set produces a model with $E_{6} \times S O(4)^{3} \times$ $U(1)^{2} \times E_{8}$ gauge group and with $(2,2)$ world-sheet supersymmetry. The realistic free fermionic models under discussion have, at the level of the NAHE set, an $S O(10)$ symmetry and only $(2,1)$ world-sheet supersymmetry. While the $(2,1)$ NAHE symmetry is further broken to $(2,0)$ by a set of non-NAHE basis vectors, the underlying $(2,2)$ 
and $(2,1)$ substructures are why we can identify the internal fermions $\{y, \omega \mid \bar{y}, \bar{\omega}\}$ with the compactified dimensions in the bosonic formulation, and the $\{\chi, \bar{\eta}\}$ with their complexified superpartners.

In these models the basis vector $2 \gamma$ replaces the vector $\mathbf{X}$. The set $\{\mathbf{1}, \mathbf{S}, \xi=$ $\left.\mathbf{1}+\mathbf{b}_{1}+\mathbf{b}_{2}+\mathbf{b}_{3}, 2 \gamma\right\}$ produces a model with $N=4$ supersymmetry and $S O(16) \times$ $S O(12) \times S O(16)$ gauge group. Alternatively, we can construct the same model by using the set $\{\mathbf{1}, \mathbf{S}, \mathbf{X}, \xi\}$. From this set we can construct two $N=4$ models which differ by the discrete choice of the free phasef

$$
c\left(\begin{array}{c}
\mathbf{X} \\
\xi
\end{array}\right)= \pm 1
$$

For one phase choice $(+)$ the gauge group is $E_{8} \times S O(12) \times E_{8}$, which is the standard toroidal compactification. This is a $(2,2) \|$ model. However, for the alternate phase choice $(-)$ the states in the spinorial representation of $S O(16)$ which make up part of the adjoint of $E_{8}$ are removed by the GSO projections. Thus, we are left with an $S O(16) \times S O(12) \times S O(16)$ gauge group and apparently $(2,1)$ world-sheet supersymmetry.

Applying the orbifold twisting to the $E_{8} \times S O(12) \times E_{8}$ model breaks one of the $E_{8}$ to $E_{6} \times U(1)^{2}$ and keeps the $(2,2)$ world-sheet supersymmetry. Applying the same orbifold twisting to the model with the second choice of phase reduces $S O(16) \times S O(12) \times S O(16)$ to $S O(10) \times U(1)^{3} \times S O(4)^{3} \times S O(16)$, similarly keeping $(2,1)$ symmetry.

Alternatively, we can start with the basis vectors that produce the $E_{6} \times U(1)^{2}$ model and then turn on the GSO projection which projects out the spinorial $\mathbf{1 6}+\overline{\mathbf{1 6}}$ in the adjoint of $E_{6}$. We will end with the same model, as the spectrum is invariant under the reordering of the GSO projections. Thus, the transition from the $(2,2)$ world-sheet supersymmetry to the $(2,1)$ world-sheet supersymmetry can be seen to be a result of a discrete choice of the free phases. This is an important observation because many of the useful simple relations that are obtained for $(2,2)$ models can be used for the realistic free fermionic models.

\footnotetext{
* In contrast, changing the the sign of the corresponding $c\left(\begin{array}{c}2 \gamma \\ \xi\end{array}\right)$ does not alter the gauge group of $\{\mathbf{1}, \mathbf{S}, \xi, 2 \gamma\}$.

${ }^{\dagger}$ More specifically the world-sheet supersymmetry is $(2,2+2+2)$.

$\ddagger$ Since $E_{8}$ is broken, the $H$-map cannot be used to justify the claim for $(2,1)$ world-sheet supersymmetry. However, unlike the $U(1)$ current of the right-moving $N=2$ world-sheet supersymmetry, the existence of the right-moving supercurrent $\bar{T}_{3 / 2}$ should be independent of choice of phases, depending only on the boundary conditions of the $\{\bar{\eta}, \bar{y}, \bar{w}\}^{i}$. Models with matching boundary conditions for all $\{\chi, \bar{\eta}\},\{y, \bar{y}\}^{i}$, and $\{w, \bar{w}\}^{i}$ pairs should retain $(2,1)$ world-sheet supersymmetry even after the right-moving $N=2 U(1)$ current is broken.
} 


\section{The origin of the anomalous $U(1)$}

To see how the anomalous $U(1)$ arises in the NAHE-based realistic free fermionic models, let us recall the $E_{6}$ model of section 2.1. This model is generated by the NAHE set basis vectors plus the vector $\mathbf{X}$ in Eq. (2.4) or alternatively by the set (2.6). Let us now consider the model with the vector $\mathbf{X}$ in Eq. (2.6) replaced with the vector $2 \gamma$. In this case we obtain a model with $S O(10) \times U(1)^{3} \times S O(4)^{3} \times S O(16)$ gauge group. The sectors $\mathbf{b}_{j}$ still produce the 24 multiplets in the $\mathbf{1 6}$ representation of $S O(10)$ with $U(1)_{j}$ charges $Q_{j}=1 / 2$. In this model the sectors $\mathbf{b}_{j}+2 \gamma$ now produce 24 multiplets in the 16 vectorial representation of the hidden $S O(16)$ gauge group, which also carry charges $Q_{i}=1 / 2, Q_{k}=1 / 2, i \neq j \neq k$. Thus, we observe that in this model $U_{1}+U_{2}+U_{3}$, which is the $U(1)$ combination (2.9) embedded in $E_{6}$ in the $E_{6}$ model, becomes the anomalous $U(1)$ combination with the total $\operatorname{Tr} Q_{A}=-576$. The anomalous $U(1)$ is therefore seen to arise in this model due to the breaking of the right-moving $N=2$ world-sheet supersymmetry.

Occurrence of a generic anomalous $\mathrm{U}(1)$ can depend on both boundary conditions and free phases. An interesting variation of an anomalous model from the basis set (2.6) can still occur with the phase choice $c\left(\begin{array}{c}\mathbf{X} \\ \xi\end{array}\right)=1$. While this generates the $E_{8} \times S O(12) \times E_{8}$ gauge group and $(2,2)$ world-sheet supersymmetry from the first four basis vectors, changing the sign of $c\left(\begin{array}{c}\mathbf{b}_{1} \\ \xi\end{array}\right)$ and/or that of $c\left(\begin{array}{c}\mathbf{b}_{2} \\ \xi\end{array}\right)$ in (2.7) from - to + produces an anomalous model when basis vectors $\mathbf{b}_{1}$ and $\mathbf{b}_{2}$ are included. In this case the final gauge group is $E_{6} \times U(1)^{2} \times S O(4)^{3} \times S O(16)$. While $U(1)_{E_{6}}$ defined in (2.9) remains non-anomalous (since it is still embedded in $E_{6}$ ), the orthogonal $U(1)^{\prime}$ and $U(1)^{\prime \prime}$ in (2.10,2.11) become anomalous.

Changing the sign of $c\left(\begin{array}{c}\mathbf{b}_{i} \\ \xi\end{array}\right)$ effectively transforms the $S O(16)$ spinor components of the $E_{8}$ gauge vectors, coming from the $\xi$-sector, into a massless matter state (the $\overline{\mathbf{1 2 8}}$ of $S O(16)$ ), thereby breaking $E_{8}$ to $S O(16)$. Simultaneously, under sign change

of $c\left(\begin{array}{c}\mathbf{b}_{i} \\ \xi\end{array}\right)$, the eight copies of the 27 rep of the observable sector $E_{6}$ (along with some states carrying only $S O(4)^{3}$ and $U(1)_{i}$ charges) originating in $\left(\mathbf{b}_{i}, \mathbf{b}_{i}+\mathbf{X}\right)$ are replaced by eight copies of a $\mathbf{1 6}$ rep of $S O(16)_{\text {hid }}$.

If only the sign of $c\left(\begin{array}{c}\mathbf{b}_{1} \\ \xi\end{array}\right)$ and not that of $c\left(\begin{array}{c}\mathbf{b}_{2} \\ \xi\end{array}\right)$ is changed (or vice versa), then the sector $\mathbf{b}_{3}=\mathbf{1}+\mathbf{b}_{1}+\mathbf{b}_{2}+\xi$ produces exactly eight copies of the $\mathbf{1 6}$ rep of $S O(16)_{\text {hid }}$ that also carry $U(1)^{\prime}$ and $U(1)^{\prime \prime}$ charge. If both signs change, then $\mathbf{b}_{3}$ yields only $S O(4)^{3} \times U(1)_{i}$ reps. In the former case (with $c\left(\begin{array}{c}\mathbf{b}_{1} \\ \xi\end{array}\right)$ transformed),

$$
\operatorname{Tr}\left(\eta_{1}, \eta_{2}, \eta_{3}\right)=(-96,192,-96)
$$

Here the anomaly can be placed entirely in $U(1)_{A} \equiv U(1)_{1}-2 U(1)_{2}+U(1)_{3}$, also leaving $U(1)^{\prime} \equiv U(1)_{1}-U(1)_{3}$ as a good symmetry. In the latter case,

$$
\begin{aligned}
\operatorname{Tr}\left(\eta_{1}, \eta_{2}, \eta_{3}\right) & =(-96,192,-96)+(192,-96,-96) \\
& =(96,96,-192),
\end{aligned}
$$


which instead leaves $U(1)^{\prime} \equiv U(1)_{1}-U(1)_{2}$ as a good symmetry and $U(1)_{A} \equiv$ $U(1)_{1}+U(1)_{2}-2 U(1)_{3}$ as the anomalous one. (2,2) world-sheet supersymmetry appears retained in both cases.

These sign changes in $c\left(\begin{array}{c}\mathbf{b}_{i} \\ \xi\end{array}\right)$ produce a trace anomaly because they destroy the delicate charge cancelation between the $\mathbf{b}_{i=1,2,3}$ sectors in the NAHE set. Relatedly, destruction of this symmetry under these sign changes, would most likely make NAHE-based $Z_{2} \times Z_{2}$ three generation models extremely difficult, if not impossible, even after addition of non-NAHE sectors.

\section{Contributions to $U(1)_{A}$ from beyond the NAHE set}

In all models discussed so far there appears a correlation between hidden sector $E_{8}$ breaking and an unembedded $U(1)$ becoming anomalous. Although broken $E_{8}$ models often suggest an anomaly will be found within a $U(1)$, the NAHE-based models demonstrate that an anomaly need not necessary appear. As counter examples we consider the model in [28] and that of [27] already discussed in section 2.2. In the first case, removing the last basis vector (the $\gamma$-like one) produces a model with

$$
S O(6) \times S O(4) \times U(1) \times S O(4) \times S O(5)^{2} \times[S O(8) \times S O(8)]_{\text {hidden }},
$$

while similarly altering the model in section 2.2 produces a gauge group

$$
S O(6) \times S O(4) \times U(1)^{2} \times\left[S U(2)_{2}\right]^{4} \times[S O(16)]_{\text {hidden }} .
$$

The single $U(1)$ in the first model and both $U(1)$ in the second model are nonanomalous even though the world-sheet supersymmetry in both models has been reduced from $(2,2)$ to $(2,1)$.

While a broken $E_{8}$ does not necessarily imply an anomaly will appear if there are any $U(1)$ 's, an unbroken level-one $E_{8}$ does imply that an anomalous $U(1)$ cannot appear. This is an example of a more general rule:

Theorem 1. A given $U(1)_{i}$ in the observable (hidden) sector is anomaly free if there is a hidden (observable) group $\mathcal{G}$ such that all massless $\mathcal{G}$-charged states have vanishing charge $Q_{i}$ [21, 29].

While $E_{8}$ has been broken in the gauge groups (4.1) and (4.2), Thm. 1 explains why none of the $U(1)$ are anomalous in either case. Prior to the appearance of a $\gamma$-sector in each model, all non-trivial reps of the respective hidden sector gauge groups do not carry additional $U(1)$ charge. In fact, without a $\gamma$-sector there are no non-trivial massless reps of $S O(8)_{\text {hid }}^{2}$ in (4.1), nor of $S O(16)_{\text {hid }}$ in (4.2), in the respective models. In both cases, adding back the $\gamma$-like basis vectors (or even just

\footnotetext{
$\S$ Henceforth, unless it specifically stated otherwise, a $U(1)$ under discussion is assumed not to be embedded in a non-Abelian gauge group.
} 
$2 \gamma$ ) generates massless states that are both hidden-sector non-singlets and that carry $U(1)$-charges.

Thm. 1 actually specifies conditions more stringent than is necessary for anomaly prevention in a $U(1)$. The necessary and sufficient condition is stated in:

Theorem 2. A given $U(1)_{i}$ is anomaly-free if there is a simple gauge group $\mathcal{G}$ in a model such that the trace of $Q_{i}$ over non-trivial massless reps of $\mathcal{G}$ is zero [21, 29].

A $U(1)$ can remain non-anomalous by reason of Thm. 2, if, for example, there is a $Z_{2}$ symmetry that transforms states with $U(1)$ charge $Q$ into states with charge $-Q$, resulting in only vector-like pairs of states with regard to that $U(1)$ [29]. Consider for instance model 2 of [26]). When basis vectors $V_{3}, V_{6}$, and $V_{7}$ (as defined in Table A.2 of [26]) are removed from this model, five anomaly-free $U(1)$ appear that demonstrate this type of anomaly cancelation. Here the gauge group is $E_{6} \times U(1)^{5} \times S O(22)$. While there are numerous massless 22's of $S O(22)$ and 27's of $E_{6}$ in the model carrying non-zero charge under the five $U(1)$, a $Z_{2}$ symmetry exists such that for every $(22$ or 27) state with $U(1)^{5}$ charge vector $\vec{Q}$ there is a respective (22 or 27) state with charge vector $-\vec{Q}$.

Both Thms. 1 and 2 rest on the fact that modular invariance of string models relates the traces of an anomalous $U(1)_{A}$ over all gauge groups in the model,

$$
\frac{1}{K_{m}} \operatorname{Tr}_{\mathcal{G}_{m}} T(R) Q_{A}=\frac{1}{3} \operatorname{Tr} Q_{A}^{3}=\operatorname{Tr} Q_{B}^{2} Q_{A}=\frac{1}{24} \operatorname{Tr} Q_{A} \equiv 8 \pi^{2} \delta_{\mathrm{GS}},
$$

(generally known as the universal Green-Schwarz (GS) relation), where $K_{m}$ is the level of the gauge group $\mathcal{G}_{m}$ and $2 T(R)$ is the index of the representation $R$. (Both anomalous charge $Q_{A}$ and the anomaly-free charges $Q_{B}$ have been rescaled so that $K_{A}=K_{B}=1$.) Since $E_{8}$ level-one has no massless matter states, an unbroken levelone $E_{8}$ in a model automatically declares all $U(1)$ anomaly-free. This relationship between traces means that if the $U(1)$ charge trace is zero over the non-trivial reps of a particular simple gauge group, the trace is zero over the non-trivial reps of all simple gauge groups!

Consideration of Thms. 1. and 2. for all $U(1)_{i}$ in a model leads to:

Theorem 3. A model is completely free of anomalous $U(1)$ if for each $U(1)_{i}$, there is at least one gauge group $\mathcal{G}$ for which (a) all non-trivial massless reps of $\mathcal{G}$ do not carry $U(1)_{i}$ charge, or (b) the trace of $Q_{i}$ over all massless non-trivial reps of $\mathcal{G}$ is zero [21, 29].

This implies that " $U(1)$-quarantined" models, defined as models with at least one hidden (observable) sector simple gauge group whose non-trivial reps carry no observable (hidden) sector $U(1)$ charge, are guaranteed to be anomaly free, independent of its world-sheet supersymmetry class.

It is an interesting question whether free fermionic " $U(1)$-quarantined" $S U(3)_{C} \times$ $S U(2)_{L} \times U(1)^{n}$ or semi-GUT models can be found. If such models exist, can they 
yield realistic phenomenology? Of particular interest would be models of this class with a modified $\gamma$ that breaks the observable group $S O(6) \times S O(4)$ to $S U(3) \times$ $S U(2) \times U(1)^{2}$ yet does not produce hidden sector states carrying observable $U(1)$ charges. NAHE class models of this type can indeed be generated. For example, instead of adding (2.14) to the NAHE set (2.2), consider the variation

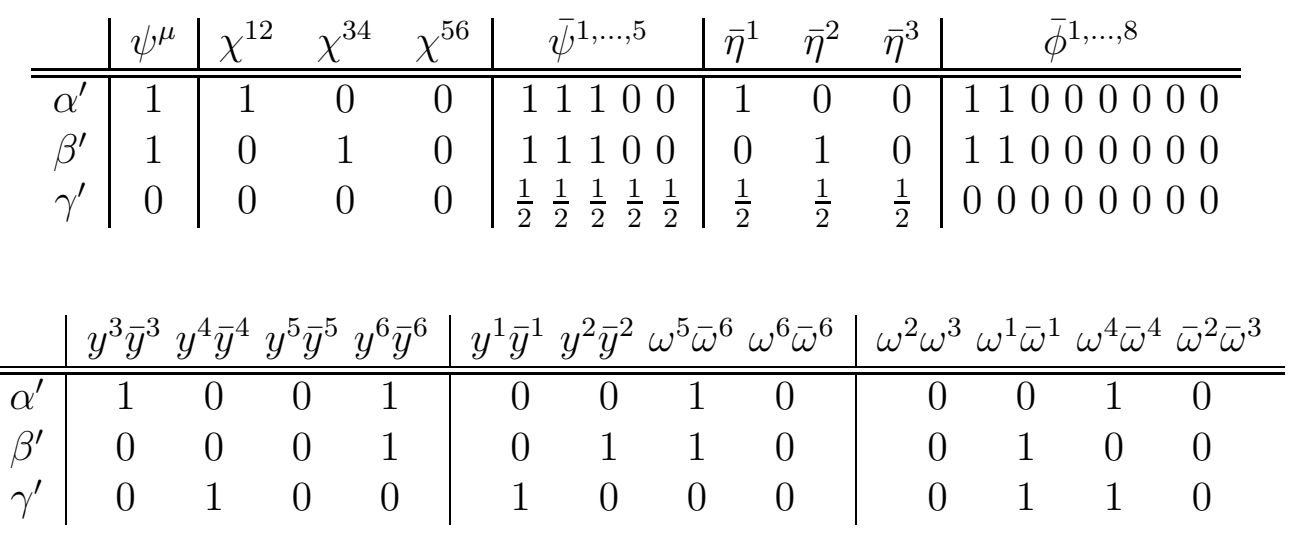

instead, with the choice of GSO projection coefficients:

$$
\begin{aligned}
c\left(\begin{array}{c}
\mathbf{b}_{i} \\
\mathbf{b}_{j}
\end{array}\right) & =c\left(\begin{array}{c}
\mathbf{b}_{i} \\
\mathbf{S}
\end{array}\right)=-c\left(\begin{array}{l}
\mathbf{S} \\
\mathbf{1}
\end{array}\right)=-c\left(\begin{array}{l}
\mathbf{1} \\
\mathbf{1}
\end{array}\right)=c\left(\begin{array}{c}
\alpha^{\prime}, \beta^{\prime}, \gamma^{\prime} \\
1
\end{array}\right)=-1 \\
-c\left(\begin{array}{c}
\alpha^{\prime}, \beta^{\prime} \\
\mathbf{S}
\end{array}\right) & =c\left(\begin{array}{c}
\gamma^{\prime} \\
\mathbf{S}
\end{array}\right)=c\left(\begin{array}{c}
\alpha^{\prime} \\
\mathbf{b}_{1}
\end{array}\right)=c\left(\begin{array}{c}
\beta^{\prime} \\
\mathbf{b}_{2}
\end{array}\right)=c\left(\begin{array}{c}
\gamma^{\prime} \\
\mathbf{b}_{i}
\end{array}\right)=-c\left(\begin{array}{c}
\alpha^{\prime} \\
\mathbf{b}_{2}
\end{array}\right)=-1 \\
c\left(\begin{array}{c}
\beta^{\prime} \\
\mathbf{b}_{1}
\end{array}\right) & =c\left(\begin{array}{c}
\alpha^{\prime}, \beta^{\prime} \\
\mathbf{b}_{3}
\end{array}\right)=-c\left(\begin{array}{l}
\beta^{\prime} \\
\alpha^{\prime}
\end{array}\right)=-c\left(\begin{array}{l}
\gamma^{\prime} \\
\beta^{\prime}
\end{array}\right)=c\left(\begin{array}{l}
\gamma^{\prime} \\
\alpha^{\prime}
\end{array}\right)=1
\end{aligned}
$$

$(\mathrm{j}=1,2,3)$, with the others specified by modular invariance and space-time supersymmetry. As usual, the $\alpha^{\prime}$ and $\beta^{\prime}$ basis vectors break $S O(10)$ to $S O(6) \times S O(4)$ and $\gamma^{\prime}$ reduces $S O(6) \times S O(4)$ to $S U(3)_{C} \times S U(2)_{L} \times U(1)^{2}$. Here, however, $\gamma^{\prime}$ does not disturb the hidden sector $E_{8}$. Instead only $\alpha^{\prime}$ and $\beta^{\prime}$ affect $E_{8}$ and break it to $E_{7} \times S U(2)$. The final observable gauge group is not $S U(3)_{C} \times S U(2)_{L} \times U(1)^{n}$ though, because $2 \gamma^{\prime}$ both enlarges $S U(3)_{C}$ to $S U(4)_{C}$ and adds an extra $S U(2)$ factor. The complete gauge group is thus,

$$
S U(4)_{C} \times S U(2)_{L} \times S U(2) \times U(1)^{4} \times\left[E_{7} \times S U(2)\right]_{\text {hidden }} .
$$

Unlike $E_{8}, E_{7}$ has a massless rep at level 1 , the $\mathbf{5 6}$. Here the only such $E_{7}$ state is also a doublet under $S U(2)_{\text {hid }}$. This state receives contributions of $\frac{3}{4}$ and $\frac{1}{4}$ to its conformal dimension from these $E_{7}$ and $S U(2)$ reps, respectively, making the state exactly massless. Hence, there is no room for it to carry a charge under any of the observable sector $U(1)_{i}$. Thus, all four of the $U(1)_{i}$ are anomaly-free by Thm. 1 ! The model is chiral with a net $6=7-1$ quark-doublet generations in $(4,2,1)$ and $(\overline{4}, 2,1)$ reps of $S U(4)_{C} \times S U(2)_{L} \times S U(2)$. 
Additional basis vectors must be added to this model to break $S U(4)_{C}$ to $S U(3)_{C}$. As long as space-time supersymmetry is kept, variations of the phase coefficients from those given in (4.6) either do not alter the gauge group in (4.7) or only break $E_{7}$ to $S O(12) \times S U(2)$. In the latter event, a $U(1)$ anomaly appears simultaneously with four hidden sector 12 reps of $S O(12)$ that carry observable sector $U(1)_{i}$ charges.

As we have see in section 3 , an important consequence of the NAHE set basis vectors is that they result in family universality of the anomalous $U(1)$ for a a suitable choice of the GSO projection coefficients. This is an outcome of the cyclic permutation symmetry which characterizes the $Z_{2} \times Z_{2}$ orbifold compactification. However, the set of basis vectors that define the three generation free fermionic models contains three additional basis vectors beyond the NAHE set, denoted typically by $\{\alpha, \beta, \gamma\}$. The immediate question is whether the additional basis vectors preserve the family universality of the anomalous $U(1)$. The answer, in general, is negative. The universality of the anomalous $U(1)$ may be spoiled from several sources. One is that the GSO projections of the additional basis vectors may project part (or all) of the $\mathbf{1 6}$ of $S O(10)$ from some of the sectors $\mathbf{b}_{j}$. Thereby the number of states from the given sector $\mathbf{b}_{j}$ will be reduced and the universality of the anomalous $U(1)$ is spoiled. However, these cases in general will not lead to three generation models and therefore are not of great interest.

Another way by which the universality of the anomalous $U(1)$ can be spoiled is due to the contribution of the massless states from the sectors which arise from the additional basis vectors. It is not apparent how to extract general patterns from these additional contributions. For our purposes here we consider, as an example, the model of ref. [28]. In this model the contribution from the NAHE set basis vectors plus the vector $2 \gamma$ (in the notation of ref. [28] this would be the sector $2 \beta$ ) contribute universally to the three $U(1)$ 's, $U_{1}, U_{2}$ and $U_{3}$, which arise from the observable $E_{8}$. Their combined contribution to these three $U(1)$ 's is $(24,24,-24)$. The contribution from the other sectors (see ref. [28] for details of the spectrum) spoils the family universality of the anomalous $U(1)$ to give $\operatorname{Tr} U_{1}=-24, \operatorname{Tr} U_{2}=-30, \operatorname{Tr} U_{3}=18$, $\operatorname{Tr} U_{5}=6, \operatorname{Tr} U_{6}=6$ and $\operatorname{Tr} U_{8}=12$. The rotated $U(1)$ symmetry containing the entire anomaly is given by (5.2). Examination of several of the other models which appear in the literature [28, 16, 26, 30, 31] show that the anomalous $U(1)$, in the generic case, is indeed not universal. It is therefore quite interesting that there exist some examples in which the anomalous $U(1)$ is family universal. The implications of the anomalous $U(1)$ in connection with supersymmetry breaking and flavor changing neutral currents will be reported in ref. [11].

\section{Phenomenological considerations}

In this section we discuss some of the phenomenological implications related to the existence of an anomalous $U(1)$ in superstring models. The existence of an anomalous $U(1)$ in superstring models has important implications on the phenomenological 
properties of the models. The anomalous $U(1)$ generates a Fayet-Iliopoulos D-term which breaks supersymmetry at the Planck scale. Supersymmetry is restored by assigning some non-vanishing VEVs to a set of Standard Model singlets in the massless string spectrum which are charged under the anomalous $U(1)$ and cancel the D-term. As in general these fields are also charged under the anomaly free $U(1)$ combinations, cancelation of the anomaly free D-term equations results in a set of non-trivial constraints on the allowed pattern of non-vanishing VEVs. In addition, F-flatness constraints impose that the superpotential and all of its derivatives vanish as well. To insure a supersymmetric vacuum, a chosen pattern of VEVs must then satisfy all the D-term and F-term flatness constraints. The mechanism by which the anomalous $U(1)$ D-term equations is canceled is often referred to in the literature as the Dine-Seiberg-Witten (DSW) mechanism [2]. The scale associated with this breaking is therefore often referred to as the DSW scale.

As we noted in the introduction, some of the phenomenological implications of the anomalous $U(1)$ symmetry, and possible patterns of VEVs, have been investigated in specific models. Most notable among those is perhaps the generation of hierarchical fermion masses. Since the allowed fermion mass terms are restricted by the Abelian and discrete symmetries in the string models, the fermion mass terms for the lighter generations are obtained from nonrenormalizable terms which are suppressed by inverse powers of the effective string scale. The relevant nonrenormalizable terms also contain fields which obtain non-vanishing VEV in the application of the DSW mechanism. Then some of the nonrenormalizable terms become effective renormalizable operators. Successive orders are suppressed by the ratio of these VEVs to the effective string scale. In this manner a hierarchical fermion mass spectrum can be generated. In this paper, however, our interest is not in a possible pattern of VEVs but rather in what we may learn by studying the $U(1)$ anomalies, which appear in specific models, and possibly the set of fields and their charges under the relevant $U(1)$ symmetries.

In general, a number of $U(1)$ symmetries may appear anomalous in the original basis in which each $U(1)_{i}$ current is generated by a single complex world sheet free fermion. Of the $n$ anomalous $U(1)$ 's we can always form $n-1$ anomaly free combinations by rotating all the anomaly into a single, unique anomalous $U(1)_{A}$, specified by

$$
U(1)_{A} \equiv k \sum_{i}\left\{\operatorname{Tr} Q_{i}\right\} U(1)_{i},
$$

where $k$ is a normalization coefficient. Obviously, all the possible combinations of anomalous $U(1)$ symmetries in the original basis, which are anomalous, must be broken at the DSW scale. Then by examining the combinations of the anomalous $U(1)$ 's which remain anomalous in (5.1) at the string scale we can learn some important lessons with regard to the final gauge group that persist after application of the DSW anomaly cancelation mechanism.

An example of this is provided in the model whose basis vectors and one-loop 
GSO phases are given in ref. [28]. With the choice of GSO projection coefficients given in ref. [28], the anomalous $U(1)$ symmetries are: $\operatorname{Tr} U_{1}=-24, \operatorname{Tr} U_{2}=-30$, $\operatorname{Tr} U_{3}=18, \operatorname{Tr} U_{5}=6, \operatorname{Tr} U_{6}=6$ and $\operatorname{Tr} U_{8}=12$. Thus, the entire anomaly can be rotated into

$$
U_{A}=-4 U_{1}-5 U_{2}+3 U_{3}+U_{5}+U_{6}+2 U_{8}
$$

Changing the phase coefficient,

$$
c\left(\begin{array}{c}
\mathbf{b}_{4} \\
\mathbf{1}
\end{array}\right)=+1 \rightarrow c\left(\begin{array}{c}
\mathbf{b}_{4} \\
\mathbf{1}
\end{array}\right)=-1,
$$

alters the anomalous $U(1)$ 's to: $\operatorname{Tr} U_{C}=-18, \operatorname{Tr} U_{L}=12, \operatorname{Tr} U_{1}=-18, \operatorname{Tr} U_{2}=-24$, $\operatorname{Tr} U_{3}=24, \operatorname{Tr} U_{4}=-12, \operatorname{Tr} U_{5}=6, \operatorname{Tr} U_{6}=6, \operatorname{Tr} U_{7}=-6, \operatorname{Tr} U_{8}=12$, and $\operatorname{Tr} U_{9}=18$. The anomalous $U(1)$, thus, transforms into

$$
U_{A}=-3 U_{C}+2 U_{L}-3 U_{1}-4 U_{2}+4 U_{3}-2 U_{4}+U_{5}+U_{6}-U_{7}+2 U_{8}+3 U_{9} .
$$

This modification has an important phenomenological implication. In the model with the phase modification, Eq. (5.3), while the weak hypercharge combination, $U_{Y}=$ $1 / 3 U_{C}+1 / 2 U_{L}$, is anomaly free, the orthogonal combination, which is embedded in $S O(10), U_{Z^{\prime}}=U_{C}-U_{L}$ is anomalous. This implies that there exist models in which this $U_{Z^{\prime}}$ must be broken near the Planck scale. Therefore, in such models the universal part of the observable gauge group, arising from the $S O(10)$ gauge group of the NAHE set, must be the Standard Model gauge group. A combination of the flavor dependent $U(1)$ 's, however, may still remain unbroken.

This simple example hinges on a different, more general and important question. Namely, in specific models given the initial set of anomalous $U(1)$ symmetries and massless particle spectrum, what is the final gauge group which is consistent with the constraints imposed by the requirement of vanishing $\mathrm{F}$ - and D-flatness constraints, and hence with the requirement of $N=1$ space-time supersymmetry at the string scale? For example, in some models it may be found that the entire four dimensional gauge group must be broken in order to satisfy the cubic level $\mathrm{F}$ - and D-flatness constraints. Such models obviously cannot yield a realistic superstring model, as the Standard Model gauge group must be broken as well. The task then is to try to classify such models in terms of the world-sheet boundary conditions, and possibly by other phenomenological properties of the models.

The question of the connection between models which allow specific types of anomalous D-term solutions and other phenomenological criteria may have important implications. One of these possible implications is in regard to the cubic level Yukawa couplings in the superstring derived standard-like models, which utilize the NAHE set. The cubic level Yukawa couplings for the quarks and leptons, in a sector $\mathbf{b}_{j}$ of a NAHE-based model, are determined by the boundary conditions in the vector $\gamma$, which breaks $S O(10) \rightarrow S U(5) \times U(1)$, according to the following rule [16]

$$
\Delta_{j}=\left|\gamma\left(U(1)_{L_{j+3}}\right)-\gamma\left(U(1)_{R_{j+3}}\right)\right| \quad(j=1,2,3)
$$




$$
=\left\{\begin{array}{l}
0 \rightarrow d_{j} Q_{j} h_{j}+e_{j} L_{j} h_{j} ; \\
1 \rightarrow u_{j} Q_{j} \bar{h}_{j}+N_{j} L_{j} \bar{h}_{j},
\end{array}\right.
$$

where $\gamma\left(U(1)_{R_{j+3}}\right), \gamma\left(U(1)_{L_{j+3}}\right)$ are the boundary conditions of the world-sheet fermionic currents that generate the $U(1)_{R_{j+3}}, U(1)_{L_{j+3}}$ symmetries, respectively. Thus, we can construct models in which both up and down type quarks obtain a cubic level mass term. On the other hand, in models with $\Delta_{1,2,3}=1$ only $+\frac{2}{3}$ charged type quarks get a cubic level Yukawa coupling. The distinction between these two types of models may in fact be correlated with the types of anomalous $U(1)$ D-term solutions. To exemplify this issue we consider the model in table (5.7).

\begin{tabular}{|c|c|c|c|c|c|c|c|c|c|}
\hline & $\psi^{\mu}$ & $\chi^{12}$ & $\chi^{34}$ & $\chi^{56}$ & $\bar{\psi}^{1, \ldots, 5}$ & $\bar{\eta}^{1}$ & $\bar{\eta}^{2}$ & $\bar{\eta}^{3}$ & $\bar{\phi}^{1, \ldots, 8}$ \\
\hline$\overline{\alpha \alpha}$ & 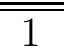 & 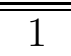 & $\overline{0}$ & $\overline{0}$ & $\begin{array}{c}11100 \\
\end{array}$ & 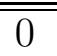 & 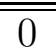 & $\overline{0}$ & $\begin{array}{llllllllll}1 & 1 & 1 & 1 & 0 & 0 & 0 & 0\end{array}$ \\
\hline$\beta$ & 1 & 0 & 1 & 0 & 11100 & 0 & 0 & 0 & 11110000 \\
\hline$\gamma$ & 1 & 0 & 0 & 1 & $\begin{array}{lllll}\frac{1}{2} & \frac{1}{2} & \frac{1}{2} & \frac{1}{2} & \frac{1}{2}\end{array}$ & $\frac{1}{2}$ & $\frac{1}{2}$ & $\frac{1}{2}$ & $\frac{1}{2} \quad 0111 \frac{1}{2} \frac{1}{2} \quad \frac{1}{2} 1$ \\
\hline
\end{tabular}

\begin{tabular}{c|cccc|cccc|cccc} 
& $y^{3} \bar{y}^{3} y^{4} \bar{y}^{4} y^{5} \bar{y}^{5} y^{6} \bar{y}^{6}$ & \multicolumn{1}{|c|}{$y^{1} \bar{y}^{1} y^{2} \bar{y}^{2} \omega^{5} \bar{\omega}^{5}$} & $\omega^{6} \bar{\omega}^{6}$ & $\omega^{2} \omega^{3} \omega^{1} \bar{\omega}^{1} \omega^{4} \bar{\omega}^{4} \bar{\omega}^{2} \bar{\omega}^{3}$ \\
\hline \hline$\alpha$ & 1 & 0 & 0 & 1 & 0 & 0 & 1 & 0 & 0 & 0 & 1 & 1 \\
$\beta$ & 0 & 0 & 0 & 1 & 0 & 1 & 1 & 0 & 0 & 1 & 0 & 1 \\
$\gamma$ & 1 & 1 & 0 & 0 & 1 & 1 & 0 & 0 & 0 & 0 & 0 & 1
\end{tabular}

The choice of generalized GSO coefficients is:

$$
c\left(\begin{array}{c}
\mathbf{b}_{1}, \mathbf{b}_{3}, \alpha, \beta, \gamma \\
\alpha
\end{array}\right)=-c\left(\begin{array}{c}
\mathbf{b}_{2} \\
\alpha
\end{array}\right)=c\left(\begin{array}{c}
\mathbf{1}, \mathbf{b}_{j}, \gamma \\
\beta
\end{array}\right)=-c\left(\begin{array}{c}
\gamma \\
\mathbf{1}, \mathbf{b}_{1}, \mathbf{b}_{2}
\end{array}\right)=c\left(\begin{array}{c}
\gamma \\
\mathbf{b}_{3}
\end{array}\right)=-1
$$

where $j=1,2,3$, The remaining coefficients are specified by modular invariance and space-time supersymmetry. By the rule (5.5) this model produces down-type Yukawa couplings from the sectors $\mathbf{b}_{1}$ and $\mathbf{b}_{2}$ and up-type Yukawa coupling from the sector $\mathbf{b}_{3}$. The model contains eight $U(1)$ symmetries, six in the observable sector and two in the hidden sector. Out of those eight, four are anomaly free and four are anomalous:

$$
\operatorname{Tr} U_{1}=18 \quad \operatorname{Tr} U_{2}=30, \quad \operatorname{Tr} U_{3}=24 \quad \operatorname{Tr} U_{4}=12 .
$$

The two $U(1)$ 's, $U(1)_{L}$ and $U(1)_{C}$, which are embedded in $S O(10)$, are anomaly free. Consequently, the weak hypercharge and the orthogonal combination, $U(1)_{Z^{\prime}}$, are anomaly free. Likewise, the two $U(1)$ 's in the hidden sector are anomaly free. The anomalous combination is given by:

$$
U_{A}=3 U_{1}+5 U_{2}+4 U_{3}+2 U_{4} \text {, with } \operatorname{Tr} Q_{A}=318 .
$$

The three anomaly-free orthogonal combinations are not unique. Different choices are related by orthogonal transformations. One choice is given by:

$$
U^{\prime}{ }_{1}=U_{1}+U_{2}-2 U_{3}
$$




$$
\begin{aligned}
U^{\prime}{ }_{2} & =U_{1}-U_{2}+U_{4} \\
U^{\prime}{ }_{3} & =3 U_{1}-U_{2}+U_{3}-4 U_{4} .
\end{aligned}
$$

The solutions to the $\mathrm{D}$ - and $\mathrm{F}$ - flatness constraints can be divided into two types. The first utilizes VEVs of fields only from the Neveu-Schwarz and the $\zeta=$ $\mathbf{b}_{1}+\mathbf{b}_{2}+\alpha+\beta$ sectors. Such solutions are stable to all orders of nonrenormalizable terms. The second allows non-vanishing VEVs for matter states from the sectors $\mathbf{b}_{j}+2 \gamma$ and for matter states which break the $U(1)_{Z^{\prime}}$ which is embedded in $S O(10)$. In the second type of solutions higher order nonrenormalizable terms will in general modify the cubic level F-flat directions. By examining the massless states from the Neveu-Schwarz and the $\zeta$ sectors, it is observed that the number of fields, with independent charges along the four D constraints is less than four in this model. The Neveu Schwarz sector produces only one field, $\Phi_{12}$. The sector $\zeta$ gives $\Phi_{45}$ and $\Phi_{3}^{ \pm}$, while $\phi_{1,2}$ and $\phi_{1,2}^{\prime}$ have the same charges, up to a multiplicative constant, as $\Phi_{12}$. However, only three of the four fields have independent charges. The complex conjugate fields can be used to relax the positive definite restriction but do not add more degrees of freedom. Thus the number of constraints is larger than the number of fields which can be used to solve them. Adding the states from the sectors $\mathbf{b}_{j}+2 \gamma$ does not resolve the problem, since they carry positive charge along the anomalous $U(1)_{A}$. Therefore, it is found that the number of independent constraints is larger than the number of flat directions, implying that solutions of the first type do not exist in this model. This example illustrates the connection between the type of D- and F-flat solutions, allowed in specific models, and other phenomenological characteristics of the models.

The next important phenomenological issue in regard to the anomalous $U(1)$ is supersymmetry breaking. As stated, the anomalous $U(1)$ generates a Fayet-Iliopoulos term that break supersymmetry near the Planck scale, and destabilizes the vacuum. Supersymmetry is restored and the vacuum is stabilized by giving VEVs to a set of standard model singlets along $\mathrm{F}$ - and D-flat directions. If these standard model singlet fields get a mass term $m$ from nonrenormalizable terms their VEV in the minimum of the potential may be shifted, resulting in a non-vanishing $\left\langle D_{A}\right\rangle$ of order $m$. This issue is discussed in detail in ref. [11. Here we would like to highlight some important properties of the anomalous $U(1)$ in this regard. Note that the $U(1)$ symmetries are in general family dependent, as they are external to the universal $S O(10)$ subgroup. Thus, in general the anomalous $U(1)$ is family dependent. This is a dangerous situation as a non-universal $\left\langle D_{A}\right\rangle$, even of the order of the electroweak scale, will give rise to non-degenerate squark masses and therefore to flavor changing neutral currents in gross excess of the experimental bounds.

We note, however, that in the free fermionic models the situation is much improved. This is a consequence of the structure of the NAHE set of boundary condition basis vectors and the underlying $Z_{2} \times Z_{2}$ orbifold. We first observe that at the level of NAHE $+2 \gamma$ model, the anomalous $U(1)$ which is the combination $U_{1}+U_{2}+U_{3}$ 
is family universal. This is a consequence of the permutation symmetry between the NAHE set basis vectors $\mathbf{b}_{1}, \mathbf{b}_{2}$, and $\mathbf{b}_{3}$, which is a reflection of the permutation symmetry between the three twisted sectors of the $Z_{2} \times Z_{2}$ orbifold. Thus, at this level the universality of the anomalous $U(1)$ is a generic result of the NAHE set basis vectors.

The realistic free fermionic models are obtained by adding several additional basis vectors to the NAHE set, which break the gauge group and reduce the number of generations to three, one from each of the sectors $\mathbf{b}_{1}, \mathbf{b}_{2}$ and $\mathbf{b}_{3}$. As we have seen above, these additional basis vectors may give rise to an additional massless spectrum which contributes to the charge trace of the anomalous $U(1)$. Therefore, the additional basis vectors may, in fact, spoil the permutation universality property which exist at the level of the NAHE set, by modifying the the traces of the individual $U(1)$ currents. Thus, the anomalous $U(1)$ combination, in terms of the $U(1)$ world-sheet currents, may be modified. In general, therefore, the resulting anomalous $U(1)$ combination will not remain family universal. Remarkably, and perhaps not surprisingly, there exist three generation models which preserve the permutation universality property of the sectors $\mathbf{b}_{1}, \mathbf{b}_{2}$ and $\mathbf{b}_{3}$, even after the additional basis vectors, beyond the NAHE set, are included. In these three generation models, therefore, the anomalous $U(1)$ is family universal. In turn we may envision that the constraints imposed by FCNC will severely restrict the allowed anomalous $U(1)$ combinations emerging from string models, and consequently the allowed boundary condition basis vectors beyond the NAHE set. In table 7.1 we show the three generation charges under the anomalous and anomaly free combinations in the model of ref. [27. As can be seen from the table, the charges of the chiral generations with respect to the anomalous $U(1)$ are indeed family universal. We also note from the table that the charges with respect to some of the other, anomaly free, $U(1)$ combinations are not family universal. One may then worry that possible non-vanishing $D$-terms of the anomaly free combinations may produce non-universal squark masses and hence FCNC. In ref. [11] this issue in investigated in detail, where it is shown that indeed such $D$-terms may arise after supersymmetry breaking, although it is often found that the non-universal contributions are suppressed relative to the universal one.

The structure exhibited by the anomalous $U(1)$ charges of the chiral generations in the model of ref. [27] is a reflection of the underlying $Z_{2} \times Z_{2}$ orbifold structure, which in this model is preserved also when the basis vectors $\{\alpha, \beta, \gamma\}$ are added to the NAHE set basis vectors. This situation is further exemplified in the model of ref. [32] where the anomalous, and anomaly free $U(1)$ combinations are

$$
\begin{aligned}
U_{A} & =U_{1}+U_{2}+U_{3} \\
U_{1}^{\prime} & =U_{1}-U_{2} \\
U_{2}^{\prime} & =U_{1}+U_{2}-2 U_{3}
\end{aligned}
$$

The anomalous $U(1)$ combination in this model is precisely the combination in the decomposition $E_{6} \rightarrow S O(10) \times U(1)$ that we encountered in considering the transi- 
tion from the $(2,2)$ models to the $(2,1)$ models in section 2 . The orthogonal anomaly free combinations are on the other hand those which are embedded in the $S U(3)$ holonomy group. In passing, it is intriguing to note that similar form of $U(1)$ combinations have recently been discussed in connection with the family mass hierarchy, from a purely phenomenological point of view [6]. Of course, the charges of the chiral generation under these $U(1)$ 's do not necessarily coincide, as those studies assume a minimal field content. Nevertheless, we may infer that fermion mass spectrum, together with the tight constraints imposed by anomaly cancelations, may yield a rather constrained structure. As we have seen, the free fermionic models, with their characteristic $Z_{2} \times Z_{2}$ orbifold structure, precisely give rise to such a structure.

\section{Conclusions}

In this paper we showed how the anomalous $U(1)$ symmetry arises in the more realistic NAHE-based free fermionic models as a consequence of the transition from the $(2,2) \rightarrow(2,0)$ models. In contrast, we also demonstrated by specific examples that general $(2,1)$ and $(2,0)$ NAHE-based models containing $U(1)$ 's can be anomaly-free under certain conditions. General constraints prohibiting appearance of anomalies were discussed. In particular, we showed that a model is guaranteed to be anomaly free when each of its $U(1)_{i}$ can be paired with at least one other gauge group, such that all the non-trivial reps of the latter group do not additionally carry a $U(1)_{i}$ charge. We then presented as an example a semi-GUT model of this type.

We discussed a simple case were the anomalous $U(1)$ is identified with the $U(1)$ in the decomposition $E_{6} \rightarrow S O(10) \times U(1)$. This simple case however has far reaching phenomenological implications. In three generation models which preserve some of the initial $Z_{2} \times Z_{2}$ orbifold structure, the $U(1) \subset E_{6}$ is, or is part of, the anomalous $U(1)_{A}$ symmetry. Consequently, in these cases the anomalous $U(1)$ is universal, and its contribution to the squark masses is family independent. The generation mass hierarchy is still generated from two sources. One is due to the non-universality of the orthogonal combinations. The other is due to the multiplicity of Higgs doublets at the string level. Recent studies have shown that combinations of anomalous and anomaly free $U(1)$ 's, similar to those that appear in the NAHE-based free fermionic models, can yield minimal parameterization of the fermion mass spectrum. These considerations provide further evidence for the potential relevance of the free fermionic models in nature.

\section{Acknowledgments}

We would like to thank Jogesh Pati, Pierre Ramond, Paul Langacker, and Mirjam Cvetič for valuable discussions. This work is supported in part by DOE Grant No. DE-FG-058ER40272. 


\section{References}

[1] For reviews and references, see e.g.,

J. Lykken, hep-ph/9511456;

J.L. Lopez, Rept. Prog. Phys. 59 (1996) 819;

K.R. Dienes, Phys. Rep. 287 (1997) 447;

A.E. Faraggi, hep-ph/9707311;

G. Cleaver, hep-th/9708023.

[2] M. Dine, N. Seiberg and E. Witten, Nucl. Phys. B289 (1987) 589;

J. Atick, L. Dixon and A. Sen, Nucl. Phys. B292 (1987) 109.

[3] I. Antoniadis, J. Ellis, J. Hagelin, and D.V. Nanopoulos, Phys. Lett. B205 (1988) 459 ;

A. Font, L.E. Ibanez, H.P. Nilles and F. Quevedo, Nucl. Phys. B307 (1988) 109; Phys. Lett. B210 (1988) 101;

J.A. Casas and C. Muñoz, Phys. Lett. B209 (1988) 214;

J.A. Casas, Katehou and C. Muñoz, Nucl. Phys. B317 (1989) 117.

[4] J.L. Lopez and D.V. Nanopoulos, Nucl. Phys. B338 (1990) 73; Phys. Lett. B251 (1990) 73;

I. Antoniadis, J. Rizos and K. Tamvakis, Phys. Lett. B278 (1992) 257;

A.E. Faraggi and E. Halyo, Nucl. Phys. B416 (1993) 63.

[5] P. Binetruy and P. Ramond, Phys. Lett. B350 (1995) 49;

E. Dudas, S. Pokorski, C.A. Savoy, Phys. Lett. B356 (1995) 45;

P. Binetruy, S. Lavignac, P. Ramond, Nucl. Phys. B477 (1996) 353;

P. Binetruy, N. Irges, S. Lavignac, P. Ramond, Phys. Lett. B403 (1997) 38.

[6] J.K. Elwood, N. Irges and P. Ramond, hep-th/9705270.

[7] P. Binutrey, S. Lavignac, S. Petcov and P. Ramond, hep-ph/9610481.

[8] L.E. Ibanez, Phys. Lett. B303 (1993) 55.

[9] A.E. Faraggi, E. Halyo, Int. J. Mod. Phys. A11 (1996) 2357.

[10] G. Dvali and A. Pomarol, Phys. Rev. Lett. 77 (1996) 3728;

P. Binutrey and E. Dudas, Phys. Lett. B389 (1996) 503;

R.N. Mohapatra and A. Riotto, Phys. Rev. D55 (1997) 4262.

[11] A.E. Faraggi and J.C. Pati, paper in preparation.

[12] J.A. Casas, J.M. Moreno, C. Munoz, M. Quiros, Nucl. Phys. B328 (1989) 272;

E.D. Stewart, Phys. Rev. D51 (1995) 6847;

E. Halyo, Phys. Lett. B387 (1996) 43;

P. Binutrey and G. Dvali, Phys. Lett. B388 (1996) 241. 
[13] J.L. Lopez and D.V. Nanopoulos, Phys. Lett. B245 (1990) 111; E. Halyo, Phys. Lett. B318 (1993) 597.

[14] T. Banks and M. Dine, Phys. Rev. D53 (1996) 5790.

[15] A.E. Faraggi and D.V. Nanopoulos, Phys. Rev. D48 (1993) 3288.

[16] A.E. Faraggi, Nucl. Phys. B387 (1992) 239; hep-ph/9708112.

[17] I. Antoniadis, J. Ellis, J. Hagelin, and D.V. Nanopoulos, Phys. Lett. B231 (1989) 65.

[18] D.J. Gross, J.A. Harvey, J.A. Martinec and R. Rohm, Phys. Rev. Lett. 54 (1985) 502; Nucl. Phys. B256 (1986) 253.

[19] I. Antoniadis, C. Bachas, and C. Kounnas, Nucl. Phys. B289 (1987) 87; H. Kawai, D.C. Lewellen, and S.H.-H. Tye, Nucl. Phys. B288 (1987) 1.

[20] For general discussions of the conditions for various numbers of left- and rightmoving world-sheet supersymmetry and related phenomenological implications see e.g. H. Braden and P. Frampton, Phys. Rev. Lett. 57 (1986) 2112; J. Lauer, D. Lust, and S. Theisen in Proceedings of the XXIV Intl. Conf. on High Energy Physics, (Springer-Verlag, Berlin, 1989).

[21] A. Schellekens, in "Superstring Construction," (North-Holland, New York, 1989) p. 2; A. Schellekens and N. Warner, Nucl. Phys. B313 (1989) 41.

[22] T. Banks and L. Dixon, Nucl. Phys. B299 (1988) 613;

S. Ferrara, D. Lüst, and S. Theisen, Nucl. Phys. B325 (1989) 501;

D. Gepner, Phys. Lett. B199 (1987) 380; Nucl. Phys. B296 (1988) 57.

[23] L. Dixon, J.A. Harvey, C. Vafa and E. Witten, Nucl. Phys. B274 (1986) 285.

[24] K.S. Narain, Phys. Lett. B169 (1986) 41;

W. Lerche, D. Lüst and A.N. Schellekens, Nucl. Phys. B287 (1987) 477.

[25] A.E. Faraggi, Phys. Lett. B326 (1994) 62.

[26] S. Chauduri, G. Hockney, and J. Lykken, Nucl. Phys. B469 (1996) 357.

[27] A.E. Faraggi, Phys. Lett. B278 (1992) 131.

[28] A.E. Faraggi, D.V. Nanopoulos, and K. Yuan, Nucl. Phys. B335 (1990) 347.

[29] T. Kobayashi and H. Nakano, INS-REP-1179; NIIG-DP-96-3; hep-th/9612066.

[30] G. Cleaver, "Aspects of CHL Three Generation String Models," in preparation. 
[31] G. Cleaver, M. Cvetič, J.R. Espinosa, L. Everett, and P. Langacker, "Classification of Flat Directions in Perturbative Heterotic Superstring Vacua with Anomalous $U(1)$," in preparation.

[32] A.E. Faraggi, Phys. Lett. B274 (1992) 47. 


\begin{tabular}{|c|c|c|rrrrrrrrr|r|r|}
\hline$F$ & SEC & $S U(3)_{C} \times S U(2)_{L}$ & $Q_{C}$ & $Q_{L}$ & $Q_{A}$ & $Q_{1}^{\prime}$ & $Q_{2}^{\prime}$ & $Q_{3}^{\prime}$ & $Q_{4}^{\prime}$ & $Q_{5}^{\prime}$ & $S U(5)_{H} \times S U(3)_{H}$ & $Q_{7}$ & $Q_{8}$ \\
\hline$L_{1}$ & $\mathbf{b}_{1}$ & $(1,2)$ & $-\frac{3}{2}$ & 0 & $\frac{1}{2}$ & $\frac{1}{2}$ & $\frac{1}{2}$ & $\frac{1}{2}$ & $\frac{1}{2}$ & $\frac{3}{2}$ & $(1,1)$ & 0 & 0 \\
\hline$Q_{1}$ & & $(3,2)$ & $\frac{1}{2}$ & 0 & $\frac{3}{2}$ & $\frac{1}{2}$ & $\frac{1}{2}$ & $-\frac{1}{2}$ & $-\frac{1}{2}$ & $-\frac{1}{2}$ & $(1,1)$ & 0 & 0 \\
\hline$d_{1}$ & & $(3,1)$ & $-\frac{1}{2}$ & -1 & $\frac{3}{2}$ & $\frac{1}{2}$ & $\frac{1}{2}$ & $-\frac{1}{2}$ & $-\frac{1}{2}$ & $-\frac{1}{2}$ & $(1,1)$ & 0 & 0 \\
\hline$N_{1}$ & & $(1,1)$ & $\frac{3}{2}$ & -1 & $\frac{3}{2}$ & $\frac{1}{2}$ & $\frac{1}{2}$ & $-\frac{1}{2}$ & $-\frac{1}{2}$ & $-\frac{1}{2}$ & $(1,1)$ & 0 & 0 \\
\hline$u_{1}$ & & $(\overline{3}, 1)$ & $-\frac{1}{2}$ & 1 & $\frac{1}{2}$ & $\frac{1}{2}$ & $\frac{1}{2}$ & $\frac{1}{2}$ & $\frac{1}{2}$ & $\frac{3}{2}$ & $(1,1)$ & 0 & 0 \\
\hline$e_{1}$ & & $(1,1)$ & $\frac{3}{2}$ & 1 & $\frac{1}{2}$ & $\frac{1}{2}$ & $\frac{1}{2}$ & $\frac{1}{2}$ & $\frac{1}{2}$ & $\frac{3}{2}$ & $(1,1)$ & 0 & 0 \\
\hline \hline$L_{2}$ & $\mathbf{b}_{2}$ & $(1,2)$ & $-\frac{3}{2}$ & 0 & $\frac{1}{2}$ & $-\frac{1}{2}$ & $\frac{1}{2}$ & $-\frac{1}{2}$ & $\frac{1}{2}$ & $\frac{3}{2}$ & $(1,1)$ & 0 & 0 \\
\hline$Q_{2}$ & & $(3,2)$ & $\frac{1}{2}$ & 0 & $\frac{3}{2}$ & $-\frac{1}{2}$ & $\frac{1}{2}$ & $\frac{1}{2}$ & $-\frac{1}{2}$ & $-\frac{1}{2}$ & $(1,1)$ & 0 & 0 \\
\hline$d_{2}$ & & $(3,1)$ & $-\frac{1}{2}$ & -1 & $\frac{3}{2}$ & $-\frac{1}{2}$ & $\frac{1}{2}$ & $\frac{1}{2}$ & $-\frac{1}{2}$ & $-\frac{1}{2}$ & $(1,1)$ & 0 & 0 \\
\hline$N_{2}$ & & $(1,1)$ & $\frac{3}{2}$ & -1 & $\frac{3}{2}$ & $-\frac{1}{2}$ & $\frac{1}{2}$ & $\frac{1}{2}$ & $-\frac{1}{2}$ & $-\frac{1}{2}$ & $(1,1)$ & 0 & 0 \\
\hline$u_{2}$ & & $(\overline{3}, 1)$ & $-\frac{1}{2}$ & 1 & $\frac{1}{2}$ & $-\frac{1}{2}$ & $\frac{1}{2}$ & $-\frac{1}{2}$ & $\frac{1}{2}$ & $\frac{3}{2}$ & $(1,1)$ & 0 & 0 \\
\hline$e_{2}$ & & $(1,1)$ & $\frac{3}{2}$ & 1 & $\frac{1}{2}$ & $-\frac{1}{2}$ & $\frac{1}{2}$ & $-\frac{1}{2}$ & $\frac{1}{2}$ & $\frac{3}{2}$ & $(1,1)$ & 0 & 0 \\
\hline \hline$L_{3}$ & $\mathbf{b}_{3}$ & $(1,2)$ & $-\frac{3}{2}$ & 0 & $\frac{1}{2}$ & 0 & -1 & 0 & -1 & $\frac{3}{2}$ & $(1,1)$ & 0 & 0 \\
\hline$Q_{3}$ & $(3,2)$ & $\frac{1}{2}$ & 0 & $\frac{3}{2}$ & 0 & -1 & 0 & 1 & $-\frac{1}{2}$ & $(1,1)$ & 0 & 0 \\
\hline$d_{3}$ & $(\overline{3}, 1)$ & $-\frac{1}{2}$ & -1 & $\frac{3}{2}$ & 0 & -1 & 0 & 1 & $-\frac{1}{2}$ & $(1,1)$ & 0 & 0 \\
\hline$N_{3}$ & $(1,1)$ & $\frac{3}{2}$ & -1 & $\frac{3}{2}$ & 0 & -1 & 0 & 1 & $-\frac{1}{2}$ & $(1,1)$ & 0 & 0 \\
\hline$u_{3}$ & & $(\overline{3}, 1)$ & $-\frac{1}{2}$ & 1 & $\frac{1}{2}$ & 0 & -1 & 0 & -1 & $\frac{3}{2}$ & $(1,1)$ & 0 & 0 \\
\hline$e_{3}$ & $(1,1)$ & $\frac{3}{2}$ & 1 & $\frac{1}{2}$ & 0 & -1 & 0 & -1 & $\frac{3}{2}$ & $(1,1)$ & 0 & 0 \\
\hline
\end{tabular}

Table 1: The three generation charges under the anomalous and anomaly free $U(1)$ combinations in the model of ref. [27]. The anomalous and anomaly free combinations are given by [27]:

$$
\begin{aligned}
& Q_{A}=2 Q_{1}+2 Q_{2}+2 Q_{3}-Q_{4}-Q_{5}-Q_{6} \\
& Q_{1}^{\prime}=Q_{1}-Q_{2} \\
& Q^{\prime}=Q_{1}+Q_{2}-2 Q_{3} \\
& Q^{\prime}{ }_{3}=Q_{4}-Q_{5} \\
& Q^{\prime}=Q_{4}+Q_{5}-2 Q_{6} \\
& Q^{\prime}{ }_{5}=Q_{1}+Q_{2}+Q_{3}+2 Q_{4}+2 Q_{5}+2 Q_{6}
\end{aligned}
$$

